\title{
Mechatronic Design of a Four-Wheel drive mobile robot and differential steering
}

\author{
Mihai Crenganis ${ }^{1, *}$, Cristina Biris $^{1}$ and Claudia Girjob $^{1}$ \\ 1 "Lucian Blaga" University of Sibiu, Engineering Faculty, MIE Department, Sibiu, Romania
}

\begin{abstract}
This paper presents, the development of an autonomous mobile robot with a four-wheel drive and differential locomotion. The mobile robot was developed in the Machines and Industrial Equipment Department from the Engineering Faculty of Sibiu. The main purpose of developing this type of mobile platform was the ability to transport different types of cargo either in industrial spaces or on rough terrain. Another important objective was that this platform could be driven in confined or tight spaces where a high degree of manoeuvrability is necessary. The great advantage of this type of mobile platform is the ability to navigate through narrow spaces due to the type of locomotion implemented. The fact that the robot has four driving wheels gives it the ability to travel on rough surfaces and easily bypass obstacles. Another great advantage of the developed mobile robot is that it has a reconfigurable structure. The drivetrain is interchangeable, it can adopt both classic wheels and Mecanum wheels. The first part of the paper presents some general aspects concerning mobile robots and two types of traction wheels used in mobile robotic structures. Subsequently, the paper presents the steps taken in the development of the mobile wheeled platform. At the end of the paper, the electronic part that will be implemented in the structure of the robot is described. The command and control of the entire mobile platform will be described in some future work.
\end{abstract}

\section{Mobile robots}

Mobile robots are complex mechatronic autonomous systems characterized by combinations of devices equipped with servo motors and sensors that can operate in a realworld or working environment. Among the mobile robots, those that move on tracks or wheels with tires are increasingly used for the execution of special works with a high degree of danger to the human operator, among which are: handling and neutralization of unexploded projectile; the execution of some corridors through the minefields; search of vehicles, trains, aircraft and buildings, followed by the tracing of explosive devices discovered in these vehicles [1-4]. One important problem for mobile robots is collision avoidance with fixed or mobile obstacles (e.g.: other mobile robots) in the robot's workspace. This can be done by several methods. Among the most commonly used

\footnotetext{
* Corresponding author: mihai.crenganis@ulbsibiu.ro
} 
navigation methods are: measuring the number of rotations made by the drive wheels, the use of accelerators and gyroscopes, electromagnetic beacons installed in the field, passive or semi-passive signals of an optical or magnetic type [5-8].

\subsection{Differential steering for mobile robots}

Differential drive or differential steering is the simplest locomotion system encountered in a mobile robot. The differential locomotion system is most often found in two-wheeled robots and consists of two wheels mounted on a common rotating axis actuated by two individual servomotors, see Figure 1 below [9-10]. The mobile robot presented in this paper also has a differential steering locomotion for classic wheel configuration so the below equations can also be used for a four-wheel drive mobile platform. This principle can be applied here because both wheels form one side have similar angular velocities.

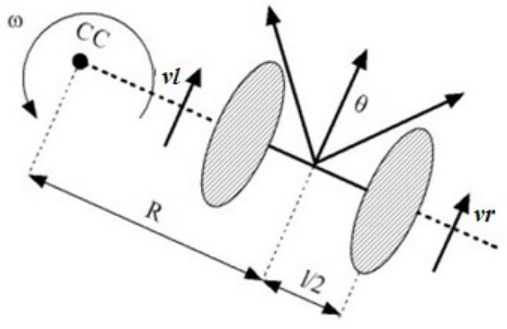

Fig. 1. Differential steering locomotion

Kinematics is that branch of classical mechanics that deals with the relationships between joint parameters or the relative movement between kinematic elements of a robot and the behaviour of the entire robotic system in the workspace or state space. With a differential steering locomotion system, a robot can realize a rotational movement around an axis perpendicular to a point contained by the axis of the two traction wheels. By changing the angular velocities of the two traction wheels, the direction of rotation can be changed. The angular velocities of the two wheels should respect the mathematical equation presented below:

$$
v_{r}=\omega \cdot(R+l / 2), v_{l}=\omega \cdot(R-l / 2) .
$$

In the above formula, $v_{1}$ characterizes the angular velocity for the left wheel and $v_{r}$ characterizes the angular velocity for the right wheel. $\mathrm{R}$ describes the radius of the circular path travelled by the center of the robot. $\omega$ the angular velocity of the wheel, and 1 the distance between the two traction wheels. The orientation of the robot is described by $\theta$ angle. By solving the system of equations one can obtain the solutions presented below:

$$
R=\left[l \cdot\left(v_{r}+v_{l}\right)\right] /\left[2 \cdot\left(v_{r}-v_{l}\right)\right], \omega=\left(v_{r}-v_{l}\right) / l
$$

If the two angular velocities are equal $\mathrm{v}_{1}=\mathrm{v}_{\mathrm{r}}$. the radius $\mathrm{R}$ is infinite. In this case, the robot will move in a straight line. If the two angular velocities have the same value but different direction $\mathrm{v}_{\mathrm{l}}=-\mathrm{v}_{\mathrm{r}}$, the distance $\mathrm{R}$ is 0 , and the robot will rotate around a vertical axis placed in the middle of the I axis. For different values for $v_{l}$ and $v_{r}$, the robot will move on a circular path of radius $\mathrm{R}$ with respect to the center of curvature $\mathrm{CC}$.

Using the right values for the angular velocities $v_{1}$ and $v_{r}$ the robot could reach a multitude of points other than the starting point. Using the direct kinematics equations one can determine the exact position of the mobile robot. 


$$
\begin{gathered}
x(t)=1 / 2 \cdot \int_{0-\mathrm{t}}\left[v_{r}(t)+v_{l}(t)\right] \cdot \cos [\theta(t)] \cdot d t \\
y(t)=1 / 2 \cdot \int_{0-\mathrm{t}}\left[v_{r}(t)+v_{l}(t)\right] \cdot \sin [\theta(t)] \cdot d t \\
{\left[\begin{array}{c}
x^{\prime} \\
y^{\prime} \\
\theta^{\prime}
\end{array}\right]=\left[\begin{array}{ccc}
\cos (\omega \delta t) & -\sin (\omega \delta t) & 0 \\
\sin (\omega \delta t) & \cos (\omega \delta t) & 0 \\
0 & 0 & 1
\end{array}\right]\left[\begin{array}{c}
x-x_{c c} \\
y-y_{c c} \\
\theta
\end{array}\right]+\left[\begin{array}{c}
x_{c c} \\
y_{c c} \\
\theta \delta t
\end{array}\right]}
\end{gathered}
$$

The above equations describe the rotational motion of a robot at a distance $\mathrm{R}$ between the middle of the axis of the two wheels and the center of curvature $\mathrm{CC}$ with angular velocity $\omega$. By integrating the above equation, starting from a set of initial conditions $\left(\mathrm{x}_{0}\right.$, $\left.\mathrm{y}_{0}, \theta_{0}\right)$, the position of the robot at a time $\mathrm{t}$ can be determined, based on the control parameters $\mathrm{v}_{1}(\mathrm{t})$ and $\mathrm{v}_{2}(\mathrm{t})$.

$$
\theta(t)=\frac{1}{2} \int_{0}^{t}\left[v_{r}(t)-v_{l}(t)\right] d t
$$

To determine the parameters, the angular velocities $\mathrm{v}_{\mathrm{l}}$, and $\mathrm{v}_{\mathrm{r}}$, so that the robot could reach a certain point, one must use the inverse kinematic equations of the robot. Because solving the above system of equations is difficult, two special cases for robot motion, with differential locomotion systems are proposed.

For the first method, if $\mathrm{v}_{\mathrm{l}}$ is equal to $\mathrm{v}_{\mathrm{r}}=\mathrm{v}$, the above equation becomes:

$$
\left(\begin{array}{l}
x^{\prime} \\
y^{\prime} \\
\theta^{\prime}
\end{array}\right)=\left(\begin{array}{c}
x+v \cdot \cos (\theta) \delta \mathrm{t} \\
y+v \cdot \sin (\theta) \delta \mathrm{t} \\
\theta
\end{array}\right)
$$

Were $x^{\prime}, y^{\prime}$ and $\theta$ ' represent the absolute velocities of the robot in the XOY coordinate system. Thus, to move a robot from $\left(\mathrm{x}_{\mathrm{S}}, \mathrm{y}_{\mathrm{S}}, \theta_{\mathrm{s}}\right)$ to $\left(\mathrm{x}_{\mathrm{g}}, \mathrm{y}_{\mathrm{g}}, \theta_{\mathrm{g}}\right)$, with $\theta_{\mathrm{g}} \neq \theta_{\mathrm{S}}$, the second control law $\left(\mathrm{v}_{1}=-\mathrm{v}_{\mathrm{r}}\right)$ can be used until $\theta_{\mathrm{g}}=\theta_{\mathrm{S}}$, then move the robot using the first control law $\left(\mathrm{v}_{\mathrm{l}}=\mathrm{v}_{\mathrm{r}}\right)$. In the second case, if $\mathrm{v}_{\mathrm{l}}=-\mathrm{v}_{\mathrm{r}}=\mathrm{v}$ is chosen, the equation becomes:

$$
\left(\begin{array}{l}
x^{\prime} \\
y^{\prime} \\
\theta^{\prime}
\end{array}\right)=\left(\begin{array}{c}
x \\
y \\
\theta+2 \frac{v \delta t}{l}
\end{array}\right)
$$

\subsection{Conventional wheel}

Conventional wheels are used because of their simplicity and because they are available in all sizes and shapes. Conventional wheels, Figure 2, have large load capacities and high tolerance to contact surface irregularities. The most common possibilities for making robots are those with differential locomotion and steering wheels. Mobile robots based on these principles have at least two wheels, each with a motor. These mobile robots can move in any direction, in any configuration. A major disadvantage of conventional wheels is the high friction that occurs over time because in differential steering situations the wheel is rotated around the vertical axis. This disadvantage reduces positioning accuracy and increases energy consumption and tire wear. This problem occurs since the wheel generates much more frictional forces, mainly when rotated about the vertical axis rather than when rotated about the central axis. The problem can be reduced by using the dual wheel system similar to the one used at the front landing gear of the aircraft [7-8]. 


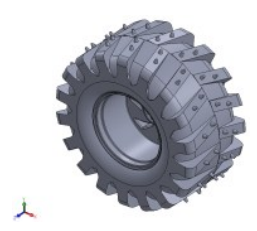

Fig. 2. Conventional wheel.

\subsection{Mecanum wheel}

The Mecanum wheel is a special design wheel developed in 1973 and it is composed of a classic wheel rim, with a different number of outer rollers, a function of its size. Using four such wheels one can get omnidirectional movement for a robot without having a conventional steering system. The working principle of a Mecanum wheel provides numerous advantages like compact and robust design, high payload values for the robot. In terms of disadvantages, they include intermittent runways and interrupted contact, low accuracy due to surface irregularities, and complex design which leads to difficulties in manufacturing. The Mecanum wheel is largely used in mobile robotic applications that require high manoeuvrability in tight spaces. This type of omnidirectional wheel has three degrees of mobility (DOF). The 3 DOF are 1st DOF wheel rotation, 2nd DOF roller rotation, and 3rd DOF rotation with sliding around the perpendicular axis from the contact point (surface - roller). In most cases, the Mecanum wheels are mounted relative to the robot body. The direction for this type of mobile robot is obtained by differing or combining the wheel's velocities. The wheels presented in Figure 3 are general Mecanum wheels. The locomotion principle for this robot is presented in Figure 4. [7-11].

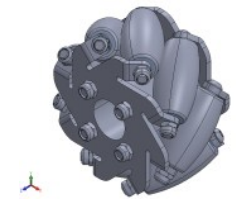

Fig. 3. Mecanum wheel.

a)

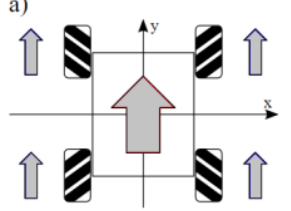

d)

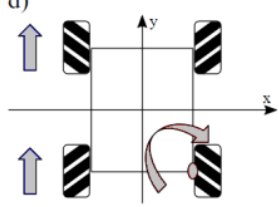

b)

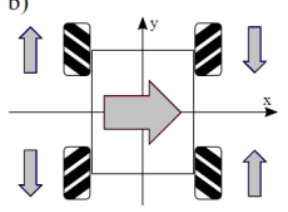

e)

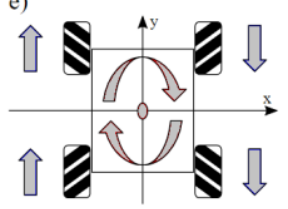

c)

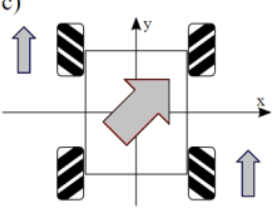

f)

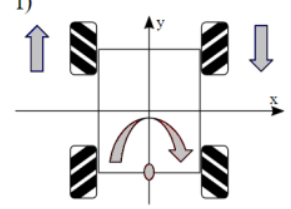

Fig. 4. Movements of a Mecanum wheel driven robot to any directions: side arrows indicate wheel drive direction; central arrow indicates vehicle moving direction a) Moving forward, b) Moving left-right, c) Moving $45^{\circ}$, d) Moving around a corner, e) Rotation around the central point the robot, f) Rotation around the middle point of one axle. 
One can observe that the roller axes make an angle of inclination with the plane of the wheel of $45^{\circ}$. Several different types of such wheels are available depending on the rollers used and their angle of inclination. Each Mecanum wheel is a self-driven traction wheel with its own actuating system. When the wheel rotates it generates a traction force perpendicular to the rotating axis, which can be decomposed into a longitudinal component and transverse component in relation to the mobile robot base. For a linear motion of the robot, all four wheels must rotate in the same direction with the same velocity. For rotation around the central vertical axis of the robot, both wheels from one side must rotate in one direction, while on the other side the wheels must rotate in the opposite direction, and with the same speed. For lateral robot movement, the diagonal wheels must rotate in one direction, while the wheels from the other diagonal must rotate in the opposite direction. A combination of differential wheel movements will allow the vehicle to move in any direction, with any rotation.

\section{Computing the necessary motor torque for driving the robot}

When considering the construction of a mobile robot, choosing an electric motor for locomotion is one of the most important decisions. Some aspects regarding the sizing of DC motors for the locomotion part of the four-wheeled mobile robot are presented below. Before choosing the DC motors, several aspects or characteristics of the mobile robot must be taken into account, namely: the dimensions of the robot, what weight it acquires, what speed and acceleration are necessary to move the robot, and last but not least the type of rolling surface. The supply voltage available for the motors must also be taken into account. To pre-dimension the traction part and choosing the type of electric motor required for locomotion, the forces acting on the robot in motion on a certain surface must be determined. It can be deduced that the higher the mass of the robot, the stronger the electric motors must be, because of higher resistance forces. During the movement of the robot on a surface, it must overcome the frictional forces between the wheels and the running surface, the frictional forces inside the motor, the inertial forces. When running on an inclined plane, the force of gravity must be considered. In this calculus, the inertia moment of the wheels is not taken into consideration because of the lower mass of the wheels and small moving velocities. The following are the steps taken to determine the speed and torque required for the electric motors. To maintain a constant speed, the electric motor must develop a motor torque that overcomes the resistive moment from the motor shaft. If the motor torque is less than the resistive torque, the robot will be at rest and this may lead to burning the winding of the electric motor if it is still powered. Figure 5 schematically represents the forces acting on the robot in the most general case. The wheels moment of inertias was not taken into account: 


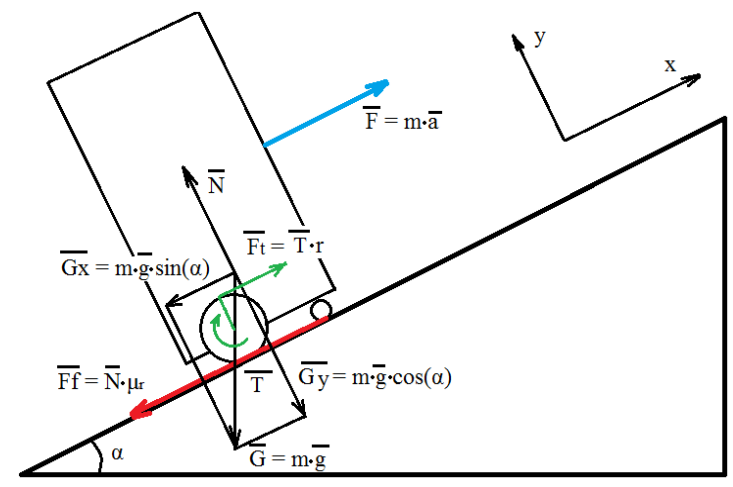

Fig. 5. Major forces acting on a mobile robot rolling on an inclined plane.

In order to determine the motor torque for actuating the robot, the resistance forces that oppose the robot movement must be calculated. Thus, the following resistive forces will be determined:

The friction force has the following expression:

$$
F f=\mu r \cdot N
$$

were:

- $\mu r$ - represents the rolling friction coefficient between the wheel and concrete 0.01 ;

- $N$ - represents the normal force on the surface:

$$
N=G y=m \cdot g \cdot \cos (\alpha)
$$

were:

- $m$ - represents the mass of the robot, $10[\mathrm{Kg}]$;

- $g$ - is the gravitational acceleration, $9.81[\mathrm{~m} / \mathrm{s} 2]$;

$-\alpha-$ is the incline angle of the rolling surface. In this case $0\left[{ }^{\circ}\right]$;

It results from the following magnitude of the friction force:

$$
F f=\mu r \cdot m \cdot g \cdot \cos (\alpha)=0.01 \cdot 10 \cdot 9.81 \cdot 1=0.981 \quad[\mathrm{~N}]
$$

The $O X$ component of the Gravity force $G$ is:

$$
G x=m \cdot g \cdot \sin (\alpha)=10 \cdot 9.81 \cdot 0=0[\mathrm{~N}]
$$

The inertia of the robot is computed using the formula:

$$
F i=m \cdot a=10 \cdot 2=20[\mathrm{~N}]
$$

were:

- $a$ - represents the maximum acceleration of the robot $2\left[\mathrm{~m} / \mathrm{s}^{2}\right]$;

After the resistant forces acting on the mobile robot were determined, the traction force necessary to drive the robot is computed using the following expression:

$$
F t>F i+G x+F f>20+0+0.981>21[\mathrm{~N}]
$$

Moreover, the traction force has the following formula:

$$
F t=T / r=21[\mathrm{~N}]
$$


were:

- $T$ - represents the motor torque;

$-\mathrm{r}-$ is the wheel radius $45[\mathrm{~mm}]=0.045[\mathrm{~m}]$.

The motor torque for driving the robot has the following expression:

$$
T=(F t \cdot r) / 2=21 \cdot 0.045 / 2=0.945 / 2=0.4725[\mathrm{Nm}]
$$

The angular velocity of the motor shaft is determined using the below formula. The maximum linear speed of the robot was limited to $0.9[\mathrm{~m} / \mathrm{s}]$ :

$$
\omega=v / r=0.9 / 0.045=20[\mathrm{rad} / \mathrm{s}]
$$

From the above relation the shaft speed is: $\mathrm{n}=190$ [rpm].

After the determination of the motor torque and the shaft speed of the electric motor, the required mechanical power to drive the mobile robot is:

$$
P=T \cdot \omega=0.4725 \cdot 20=9.45[\mathrm{~W}]
$$

\section{Hardware structure of the mobile robot}

After computing the mechanical power necessary to operate the mobile robot, four $12 \mathrm{~V}$ 37D mm DC gearmotors were chosen for the locomotion part. This type of DC motor has a cylindrical gearbox attached to the motor shaft, with straight teeth and a transmission ratio of 50:1. In Figure 6 a 12V 37D mm DC motor is presented.
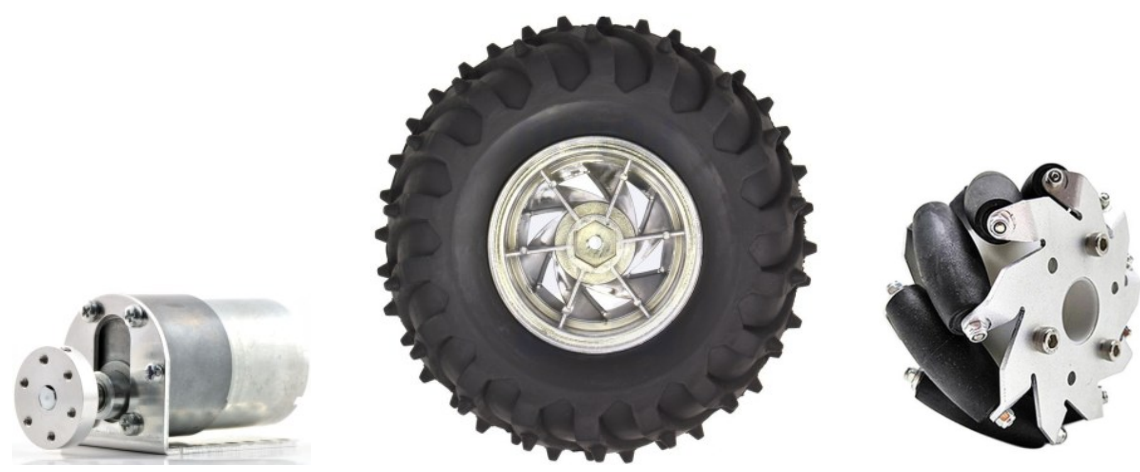

Fig. 6. 12V D37 [mm] electric DC motor for mobile robot drive and wheels used for the drivetrain.

Technical specification for the $12 \mathrm{~V} 37 \mathrm{D} \mathrm{mm}$ DC gearmotor: motor diameter $\mathrm{D}=37$ $[\mathrm{mm}]$, max. length $1=54[\mathrm{~mm}]$, mass $\mathrm{m}=0.2[\mathrm{~kg}]$, shaft diameter $\mathrm{d}=6[\mathrm{~mm}]$, gear reduction $\mathrm{i}=50: 1$, nominal voltage $\mathrm{U}=12[\mathrm{~V}]$, nominal speed $\mathrm{n} 0=200[\mathrm{rpm}]$, no load current $\mathrm{I} 0=300[\mathrm{~mA}]$; stall current $\operatorname{Imax}=5[\mathrm{~A}]$; $\operatorname{maximum}$ torque Tmax $=1.2[\mathrm{Nm}]$; The maximum electrical power consumed by the motor is determined using the relation:

$$
\mathrm{P}=\mathrm{U} \cdot \operatorname{Imax}=12 \cdot 5=60[\mathrm{~W}]
$$

To be able to command and control the four 12V DC motors, two double L298n H bridges were used in the electrical circuit of the robot. The L298N is a double H bridge integrated circuit used to command relatively high-voltage sources, designed to accept standard TTL logic levels for control. One can use the L298n H-bridge to control two DC motors or one stepper motor once. Each bridge can be independently activated or 
deactivated from the enable pins ENA, ENB. The module also contains a voltage stabilizer circuit $(5 \mathrm{~V})$ that allows operation at high voltages. Specifications of the H-bridge: maximum operating voltage, up to $40[\mathrm{~V}]$, maximum operating current, up to 3 [A]. For controlling one motor, one should respect these steps: ENA $=0 \mathrm{IN} 1=\mathrm{X}, \mathrm{IN} 2=\mathrm{X}$, represents STOP, ENA $=1 \mathrm{IN} 1=0$, IN2 $=0$, represents BRAKE, ENA = $1 \mathrm{IN} 1=0$, IN2 = $\mathrm{PWM}$, represents variable speed for direction 1 , ENA $=1 \mathrm{IN} 1=\mathrm{PWM}, \mathrm{IN} 2=0$, represents variable speed for direction 2, ENA $=1 \mathrm{IN} 1=1$, IN2 $=1$, represents BRAKE. To control the speed of one motor, enable pin ENA, IN1, IN2 pins should be connected to a microcontroller. To command and control the entire mobile robot a development board based on an ATMEL AT-Mega 2560 microcontroller was used in the electrical diagram of the robot, Figure 7 . The entire electrical diagram for the actuating the robot is presented in Figure 8.

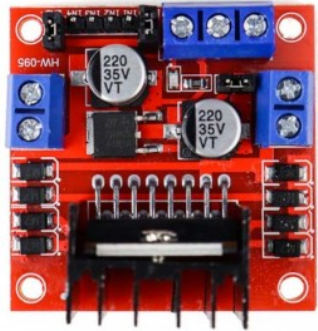

L298n Double H-bridge

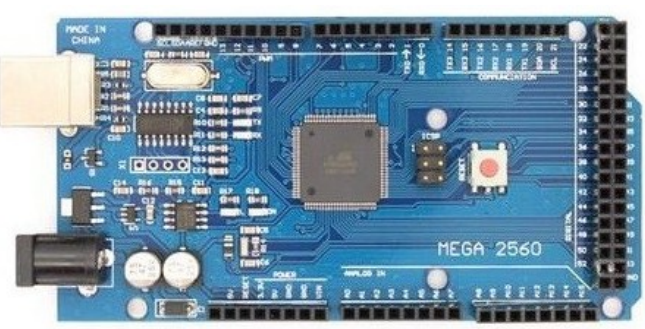

ATMEL AT_Mega 2560 microcontroller

Fig. 7. Electronic components used to command and control the mobile robot

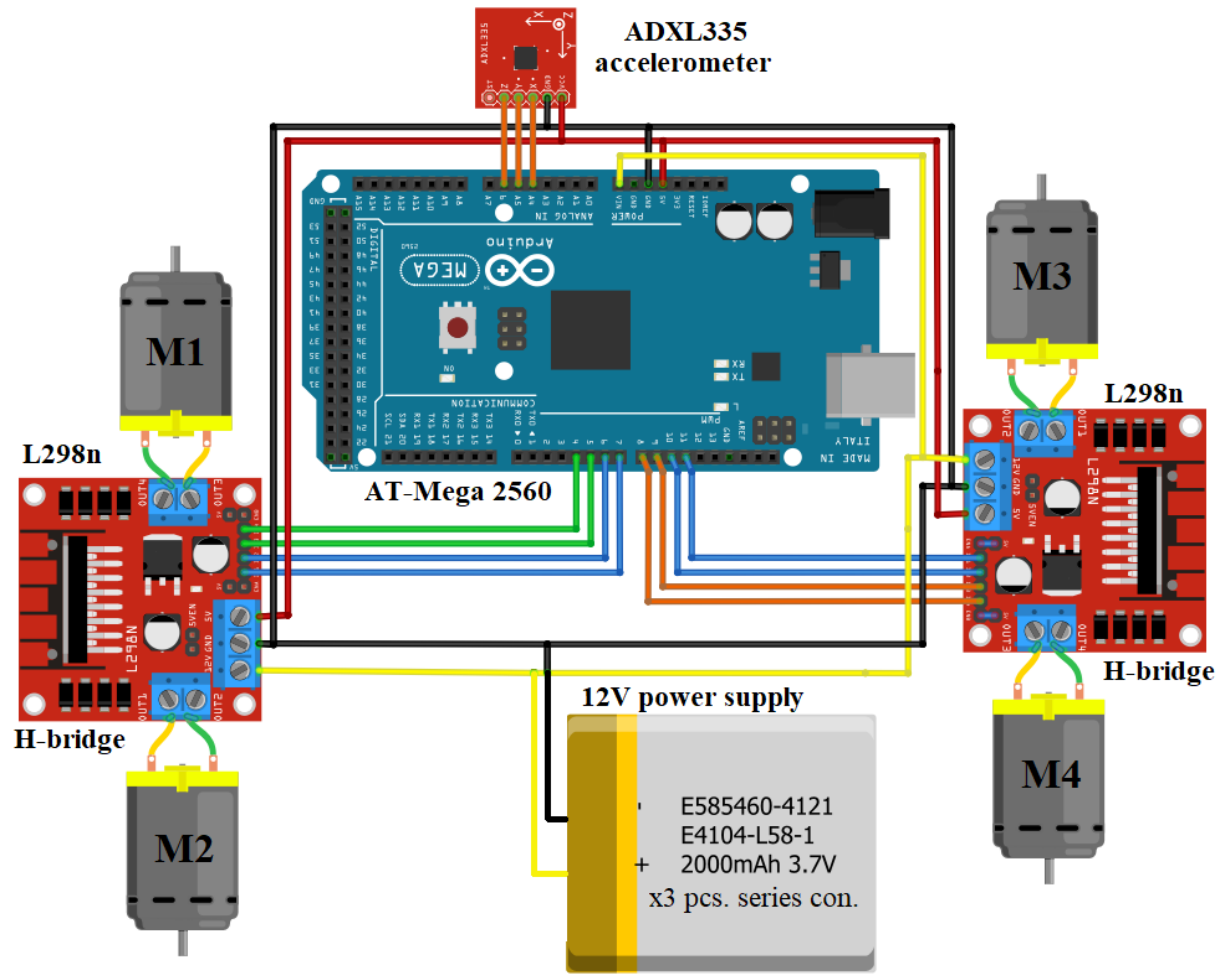

Fig. 8. Electrical diagram of the mobile robot 
The preliminary step in the realization and construction of the autonomous mobile robot was to design and model certain components of the robot structure in a 3D CAD software. This stage was necessary for a very important reason, to verify the overall dimensions of the robot and to adjust the internal components. As a result of this operation, was the ability to optimize to the maximum the space inside the robot for assembling all of the components.

In Figure 9 the entire 3D CAD model of the robot is presented. Also, below the CAD model the mechanical structure of the mobile platform is presented. In the first row one can see the mobile platform quipped with conventional wheels were as in the second colon the mobile robot equipped with Mecanum wheels is presented.
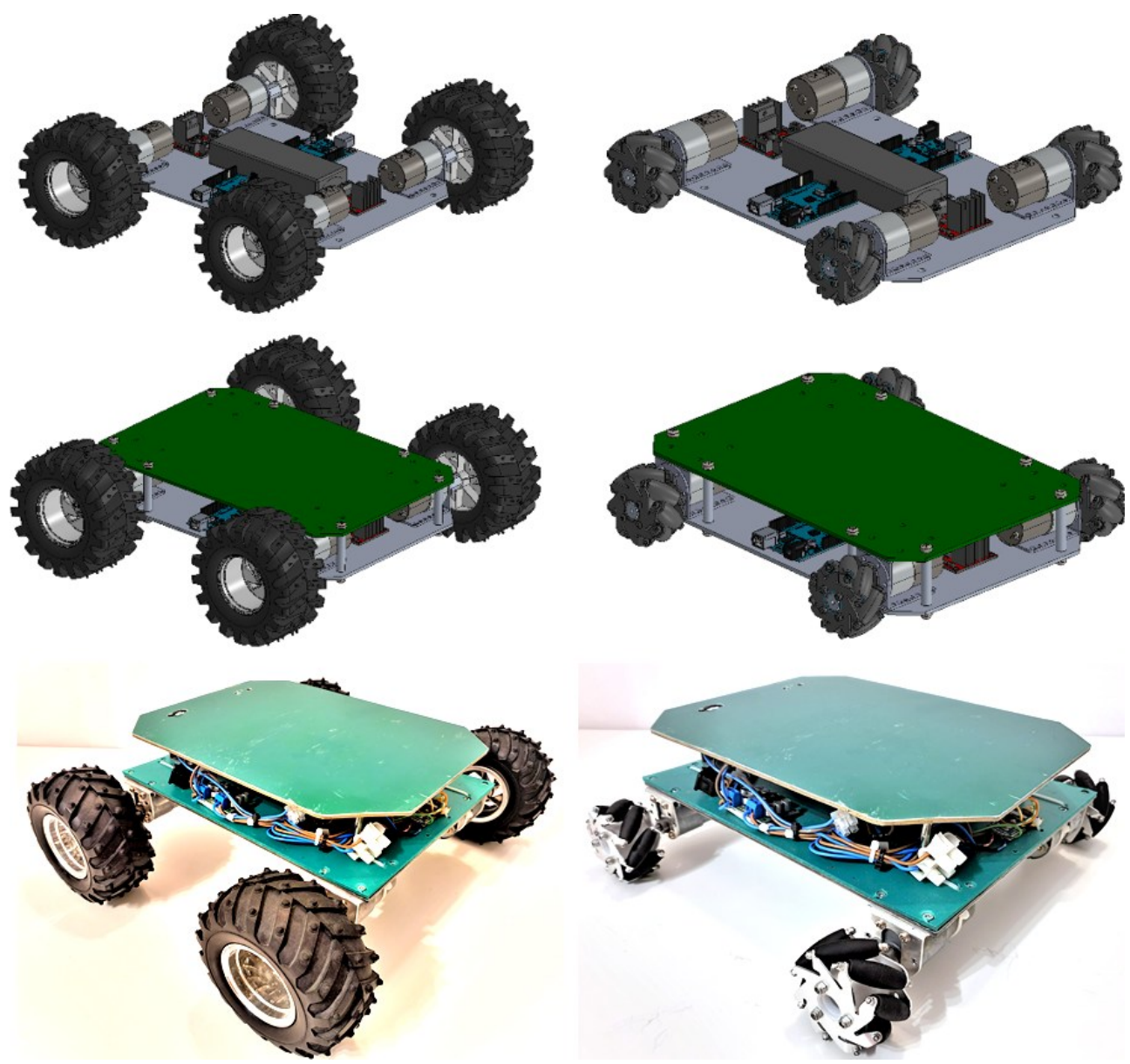

Fig. 9. Mobile, reconfigurable robot with four-wheel drive

\section{Conclusions}

As a result of the project, a mobile robot with differential steering was developed. The structure is powered by four $12 \mathrm{~V}$ powered DC motors and with a maximum power of $60 \mathrm{~W}$.

The locomotion method used to drive the robot is based on the fact that the wheels are driven independently. For the translational movement of the robot, the motor wheels must be operated in the same direction with the same velocity and in order to perform a 
rotational movement, the wheels from one side and the other must be operated in different directions of rotation or with different speeds. The advantage of this type of differential traction is that the overall dimensions of the robot can be reduced. This type of structure also offers a high possibility of changing the direction in small spaces. The biggest disadvantage is that the differential locomotion introduces certain positioning and orientation errors in the robot movement.

Another big asset of the developed mobile robot is the possibility of using interchangeable wheels. The drivetrain can use classical wheels and Mecanum wheels. The most important advantages of using classical wheels are the stability of the robot and its possibility to be driven on uneven and rough surfaces. The advantages of using Mecanum wheels is the ability of the robot to move in any direction in tight spaces without changing its orientation.

\section{Acknowledgement}

This work was supported by a grant of the Romanian Ministry of Research and Innovation, CCCDI - UEFISCDI, project number PN-III-P1-1.2-PCCDI-2017-0446/82PCCDI/201, with in PNCDI III".

\section{References}

1. J. Angeles, Fundamentals of Robotic Mechanical Systems: Theory, Methods, and Algorithms, Springer, (2006)

2. B. Siciliano, Handbook of Robotics, Springer, (2016)

3. O. Bologa, R.E. Breaz, S.G. Racz, IJCCC, 10, 803-811, (2015)

4. G. Muresan, L. Morar, R.E. Breaz, Teaching CAM Techniques and CNC Programming in Technical Universities - An Integrated Approach, (Valencia, Spain, 2014)

5. S.G. Racz, R.E. Breaz, L.I. Cioca, Evaluating Safety Systems for Machine Tools with Computer Numerical Control using Analytic Hierarchy Process, SAFETY, 5, (2019)

6. A. Marosan, G Constantin, A Chicea, et. al., A Bârsan, Acta Universitatis Cibiniensis, 72, 49-56, (2020)

7. I.A. Marosan, G Constantin, A Barsan, et. al, Creating an ethernet communication between a Simatic S7-1200 PLC and Arduino Mega for an omnidirectional mobile platform and industrial equipment, IOP Conference Series: Materials Science and Engineering, (Galati, Romania, 2020)

8. M. Crenganis, M. Tera, C. Biris, C. Girjob, ITQM 2019, Dynamic Analysis of a 7 DOF Robot Using Fuzzy Logic for Inverse Kinematics Problem, (Granada, Spain 2019)

9. O. Bologa, RE. Breaz, SG. Racz, M. Crenganis, ITQM 2016, Decision-making tool for moving from 3-axes to 5-axes CNC machine-tool, (Asan, Korea,2016)

10. M. Crenganis, R. Breaz, G. Racz, O. Bologa, ICARCV 2012, Inverse Kinematics of a 7 DOF Manipulator Using Adaptive Neuro-Fuzzy Inference Systems, (Guangzhou, China, 2012)

11. A. Marosan, G Constantin, ICMAS 2020, PID Controller Based On A Gyroscope Sensor For An Omnidirectional Mobile Platform, (Bucuresti, Romania, 2020) 